\title{
Cultural-Dialogic Interdeterminist Metaperspective for Mental Health and Well-Being
}

Vladimir A. Yanchuk

Belarus State University (Belarus), Kalvaryiskaia str. 9. 220004 Minsk, Belarus

\begin{abstract}
The static, fragmentary nature of the existing approaches to the mental health and wellbeing problems, which cannot grasp the dynamic, nonlinear, and heterogeneous nature of changes in the external natural and social environment, is stated. Author's attempt to extend the problem of this problem field vision horizons offers cultural-dialogic interdeterminist metatheory of psychological knowledge integration. Based on the determinist and indeterminist directions of scientific thought analysis is proved the epistemological heuristic potential of the cultural-dialogic interdeterminist approach. For this task solution was introduced epistemological construct "cultural-dialogic interdeterminism" allowed to analyze phenomenology in the context of quality, acquired by the whole and not reducible to the simple sum of its constituent parts. It articulated the necessity of mental health and wellbeing phenomenology multidimensional consideration in the spaces of hetero qualitative natures, psychic spheres and behavioral determinants in their cultural conditionality and qualitative specificity. This phenomenology is viewed from the heterogeneous dynamic systems approach standpoint. Theoretical and empirical explanations and evidence of cultural-dialogical interdeterminist metatheoretical approach innovativeness for the analyzed phenomenology understanding deepening is presented.
\end{abstract}

\section{Introduction}

Characterizing up to date situation in the problem field of mental health, psychiatry, and well-being L.J Kirmayer notes, mental health needs of diverse communities by integrating attention to cultural differences in knowledge, social institutions, identities, and practices. These differences affect mental health by influencing the causes and mechanisms of psychopathology, shaping illness experience and expression, and guiding processes of coping, adaptation, healing, and recovery [1]. Analysis of the problem field involves the definition of its specificity, which we see in the following. First, mental health and well-being phenomenology are not static, but dynamic; secondly, they are heterogeneous, and thirdly, they actively disseminating through migration processes intensification. This phenomenology itself is not homogeneous and static, but rather heterogeneous and dynamic. In most areas of study now exists a plethora of theories, each with its concepts, language, and underlying assumptions, but there is a lack of critical comparison, integration, and synthesis in this body of theory, resulting in conceptual clouding and ambiguity. Author's attempt to extend this problem vision horizon offers cultural-dialogic interdeterminist metatheory of mental health and well-being phenomenology integration. Based on the determinist and indeterminist directions of scientific thought analysis is proved the epistemological heuristic potential of the cultural-dialogic interdeterminist approach. For this task solution was introduced epistemological construct "cultural-dialogic interdeterminism," allowed to analyze mental health and well-being phenomenology in the context of quality, acquired by the whole and not reducible to the simple sum of its constituent parts. We articulate the necessity of mental health and well-being phenomenology multidimensional consideration in the spaces of hetero qualitative natures, psychic spheres and behavioral determinants in their cultural conditionality and qualitative specificity. Theoretical and empirical explanations and evidence of cultural dialogic interdeterminist metaperspective for mental health and well-being phenomenology are presented.
The phenomenology of mental health and well-being itself is not homogeneous and static, but heterogeneous and dynamic. In most areas of study now exists a plethora of theories, each with its own concepts, language, and underlying assumptions, but there is a lack of critical comparison, integration, and synthesis in this body of theory, resulting in conceptual clouding and ambiguity. Reflecting the current state of psychological knowledge, we can state the presence in it of an infinite number of local theories and empirical findings, leading to the drowning in the unlimited of disparate fragments, not accompanied by holistic understanding of the psychological phenomenology particularity as the integral reality of human existence that determines its functioning in it. In the framework of the proposed metatheoretical approach, an author's attempt to solve this fundamental problem, which is relevant for psychological knowledge development. Initially stated pluralistic character of modern psychological knowledge and its philosophical and epistemological grounds. Existing crisis debates in the psychological science (replicability, disintegration, empiricism, etc.) is considered in the context of deterministic and indeterministic directions of its development. I propose an interdeterministic alternative in psychological development and its innovative potential. The authors attempt to integrate the variety of existing psychological knowledge in the form of positioning in the heterogeneousmultidimensional-multiparadigm spaces of psychological knowledge in the form of three interconnected four-dimensional continua, allowing to integrate all the existing traditions and approaches

"Corresponding Author: Prof. Vladimir A. Yanchuk, Vladimir Yanchuk, Nekrasova 20, 220040 Minsk, Belarus, Tel: +375-29-754-5200; E-mail: yanchuk1954@gmail.com

Citation: Yanchuk VA (2019) Cultural-Dialogic Interdeterminist Metaperspective for Mental Health and Well-Being. Int J Psychol Behav Anal 5: 160. doi: https://doi. org/10.15344/2455-3867/2019/160

Copyright: (c) 2019 Yanchuk. This is an open-access article distributed under the terms of the Creative Commons Attribution License, which permits unrestricted use, distribution, and reproduction in any medium, provided the original author and source are credited. 
diversity is proposed the mechanism of heteroqualitative natures, psychic spheres, and behavior determinants interaction- culturaldialogic interdetermination, focusing attention on acquiring a whole new quality, not reducible to the simple sum of its constituent parts. A cultural-dialogical interdeterminist solution of the psychological knowledge integration is unfolding. Are demonstrated applied possibilities of metatheory and prospects of its development in the fields of healthcare and wellbeing?.

\section{Materials and Methods}

The primary research method is the theoretical analysis of an existing psychological broad range of knowledge, accumulated within the framework of different paradigm coordinates, traditions, and research fields. This analysis was carried out using the author's integrative-eclectic approach for psychological phenomenology analysis, which justifies the efficiency of multiplicity of sources application [2]. Within the approach, has been introduced mechanism of interparadigm dialogue, which makes it possible to find consistent meanings in the applied conceptual apparatus and research methods [3]. As an alternative to traditional logic or/or is proposed dialogical logic and/and. The analysis of ontological-epistemological foundations of existing psychological knowledge diversity was carried out using author's epistemological constructs "cultural-scientific tradition [4] and "cultural-dialogic interdeterminism [5]. With their mediation, was analyzed humanity worldview evolution during different epochs and changes in the historical, cultural, natural and technogenic contexts. As a result of the analysis, limitations of determinist and indeterminist directions of development were shown. As an alternative, a cultural-dialogic interdeterminism was proposed, the detailed description of which is presented in the author's publication $[6,7]$. Within the theoretical framework, conceptual and instrumental tools for the phenomenology of mental health and wellbeing analysis are proposed.

\section{Results}

One of the most apparent characteristics of the psychological knowledge current state is its multivoicedness, manifested in the autonomous co-existence of various theoretical traditions, approaches, and infinity of empirical correlations of the presence or absence of the infinity of the characteristics of various local psychological phenomena interrelationships. It received its official designation as psychological pluralism. Pluralism of psychological knowledge finds increased proponents [8-16]. As B.L. Paterson notes, "the goal of theoretical pluralism is not to produce a single theory that explains all aspects and attributes of a phenomenon; rather, it is intended to integrate and organize alternative theoretical perspectives in order to develop a broader perspective about the phenomenon of interest than is possible with a single theory" [17].

Articulated pluralistic tendency in the development of social knowledge, in general, and psychological knowledge I am pointing out that it is a natural consequence of the society worldview evolution throughout humanity history. The analysis of this evolution singularity was carried out through the introduced theoretical construct "cultural-scientific tradition," which allows tracing the changes taking place in the ideas of the society about the world order [6]. Cultural scientific tradition is defined as multivocal and dynamically mobile complex of philosophical, epistemological, scientific-theoretical, and emotional-aesthetic representations depending on the historical, social, and national context ...characteristic of the certain mentality, particular way of world perception, attitude, and evaluation both the cognitive capabilities of person and his place and role in the world around him. The application of this construct allowed us to trace the dynamics of the worldview various traditions (cultural syncretism, theocentrism, anthropocentrism, modernism, postmodernism) foundations, supplementing them with the tradition of dialogism, which found its supreme embodiment in dialogue as condition, mechanism and propulsion of culture and science in conditions of diversity. The general characteristic of the main cultural-scientific traditions is presented in Figure 1.

On the example of the changes that have occurred throughout the mankind history, the progressive movement from fragmentary, fetishized worldview is shown, caused by the extreme lack of knowledge about the nature and essence of the observed, leading to the objects of the surrounding world (hylozoism) animation and

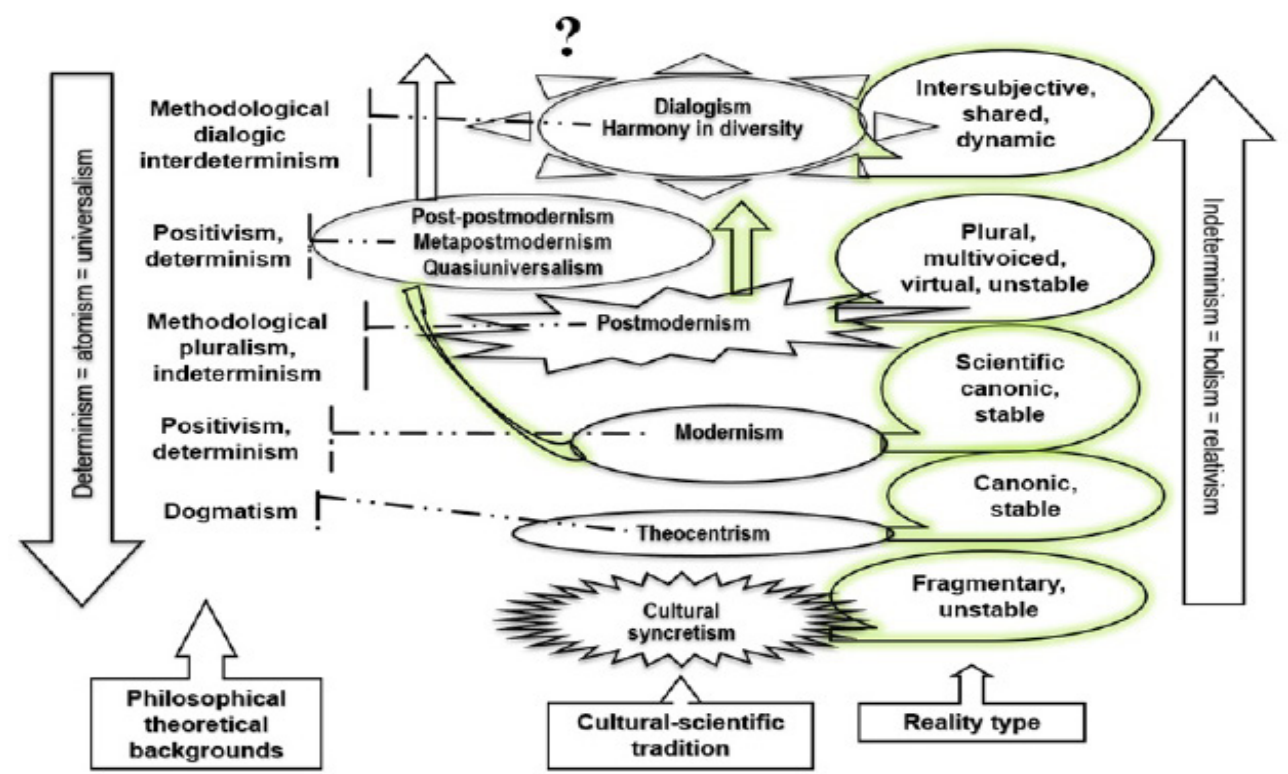

Figure 1: The evolution of the cultural-scientific traditions. 
deification to more systematic, person-centered knowledge initially oriented toward finding universal (objective) laws of the world order, and then, confronted with the problem of the any knowledge based on an unchanging universal basis innovative and heuristic resources potential exhaustibility, coming to the idea of the necessity and productivity of its diversity, polyphony in its comprehension, multiparadigmality and polyvariance.

From Figure 1 it is evident that the primary debate regarding the direction of the mental health and wellbeing phenomenology analysis development is an epistemological nature and is reduced to competition of two directions: Aristotle's atomism, oriented to the psychic primary atoms discovery in the form of behavior or psyche functioning universal laws and Galilean holism, recognizing the infinity of interrelated and interdependent psychic functioning elements. Aristotle's atomism was unsuccessful in finding the first atoms of which one can build an integral building of the psychic understanding, Galilean holism - in the impossibility of the immense infinity of psychological phenomenology embracing.

At the epistemological level, the dichotomy of atomism-holism reduces to the determinism-indeterminism dichotomy. In the deep philosophical sense today, it is entirely justified to speak about the apparent opposition of determinism and indeterminism as its antithesis. It should be emphasized that the apparent advantage of the former over the second in positivistically oriented empirical studies. A simple analysis of the publications presented in scientific journals shows the apparent dominance of empirical studies of a wide variety of mental health and wellbeing phenomenology aspects. Speaking about the crisis tendencies in psychology, S. Lilienfeld, articulates number of possible negative consequences of this crisis in discussed problem field: "(a) incentives for engaging in questionable research practices, (b) a single-minded focus on programmatic research, (c) intellectual hyperspecialization, (d) disincentives for conducting direct replications, (e) stifling of creativity and intellectual risk taking, (f) researchers promising more than they can deliver, and (g) diminished time for thinking deeply. Preregistration should assist with (a) but will do little about (b) through (g). Psychology is beginning to the right the ship, but it will need to confront the increasingly deleterious impact of the grant culture on scientific inquiry" [18].

An alternative to determinism is indeterminism, which emphasizes free will and freedom of choice as not determined by antecedent causes, that not all events have causal reasons. A fundamental analysis of the role of indeterminism in psychological and behavioral development is presented in the paper "Dynamics and Indeterminism in Developmental and Social Processes" [19]. In this paper, the indeterminist approach appears in the historical, philosophical and theoretical aspects in the context of the dynamic systems approach, which later became the subject of the same fundamental analysis in the perspective of the methodology of dynamic processes in the social and developmental sciences [20].

In an epistemological aspect, today it is possible to speak about the apparent competition between determinism and indeterminism as its antitheses. It should be emphasized that the apparent advantage of the former in positivistically oriented empirical studies. However, the recognition of the minimal heuristic possibilities of empiricism and its inability to act as a basis for complex mental health and wellbeing phenomena understanding led to a trend towards the growing popularity of the indeterministic direction. First, the indeterminism is associated with the postmodern psychological tradition, standing on positions of anti-universalism, and proclaiming multivoicedness as the mechanism and resource for overcoming the limitation of universalism in the knowledge about mental health and wellbeing phenomena. However, multivoicedness have an essential weakness it leads to discordance and loss of definiteness in studied phenomena interpretation owing to lack of the agreed and conventional meanings and accepted mechanism of their negotiation. In the deterministic approach, such a mechanism is evident and unchanged throughout the history of its existence - operationalization and verification. Thus, strengths and weaknesses are present in both directions. The solution is traditionally in the middle. In the discussed context, very suggestive is P. van Geert, one of the leading specialists in the problem field, inference: "A deterministic universe is dead because it has no degrees of freedom: Everything is predestined, and no information is created because all information is contained in the initial state (whatever that may be). An indeterministic universe is lifeless because it contains an infinite number of degrees of freedom. No information is created because every event has a similar probability. However, where the two principles meet, information and order are created in the form of highly reduced degrees of freedom, in which differences between events become meaningful and informative. The new concept of complex order, emerging out of self-organization. Fora complex order to emerge, both determinism and indeterminism are needed" [21]. There is nothing to add to this!

Positioning in the heterogeneous-multidimensional-multiparadigm spaces of psychological knowledge. The author's attempt to solve the problem of existing knowledge diversity integrating complex task, accumulated in the existing variety of paradigm coordinates, directions, traditions, and approaches, is presented within the framework of the cultural-dialogic interdeterminist metatheory [4-6].

The most challenging task is to find the bases for ordering the infinity of knowledge in the field of mental health and wellbeing phenomenology, connected with the person being in the conditions of the social environment. First, this being is multifaceted. Secondly, it is actualized in the complex interaction of the biological, psychic, and symbolic hetero qualitative natures. Moreover, we must consider that a purely mental component outside the context of the biological substrate can be isolated only in abstraction. Thirdly, the social nature itself is multifaceted in its representation in the multicultural context, which introduces an aspect of the pronounced cultural differences invisible presence, which cannot be ignored either in the field of mental health or the fields of sociology and biology. Fourthly, on what concretely or subjectedly based when trying to inventory infinity and a variety of existing in the problem field knowledge.

Finding mutually acceptable decisions in the conditions of the existing diversity is an extremely complex task in which solution is possible only in the process and using dialogue [3]. According to cultural-dialogic interdeterminist metatheory consideration of the mental health and wellbeing phenomenology must be carried out in the integrated space of existing knowledge, accumulated in different systems of paradigm coordinates. In this context, is distinguished three four-dimensional spaces allocated on nature's hetero qualitativity criteria, psychic spheres, and behavior determinants, integrating the basis of which is cultural conditioning. By continuums of mental health and wellbeing phenomena's interdeterminants spaces is understood the whole complex of internal-external reasons, interaction and mutual influence which determines the behavior uniqueness. The hetero qualitative, multidimensional and multiparadigm knowledge 
is represented in the form of the following four-dimensional continuums: by criteria of nature's hetero qualitativity: biologicalpsychic-social-culturally conditioned; by criteria of psychic spheres: conscious-unconscious-existential-culturally conditioned; by criteria of behaviour determinants: personality-environment -activityculturally conditioned. Dedicated spaces are analyzed in the context of their cultural conditioning, serving as a common universal interdeterminants.

However, these spaces do not exist as autonomous, self-sufficient entities but as complementary and mutually expanding opportunities and analyzed phenomena's comprehension depth. As combined, they describe human psychic specificity in its bio-psycho-social essences, functioning in spheres of the conscious-unconsciousexistential, and manifested in personality, environment, and activity aspects, culturally conditioned in all diversity of its emic and ethic aspects. Today is evident, that action and experience are understood as embodied (occurring in a body as opposed to just in the brain), embedded (within a social context), enacted (through interaction with the world), and extended (reaching beyond the boundaries of the physical body to include aspects of the world in the process of cognition) [22].

The presented continua allow us to include in the analysis an achievement that has recently received wide acceptance, including in biomedical sciences, bio-psycho-social approach, psychoanalytic and existential-phenomenological approach whose contribution to the psychological knowledge development is evident, finally, the traditional research fields, articulated by Kurt Levin in his famous formula $B=f(P, E)$.

An even more full range of approaches represents the paradigmatic variety: behaviorist and its modern development in the form of cognitive-behavioral modification; Interactionist; psychoanalytic; cognitive; existential-phenomenological; activity and others. It should be noted that most of the ideological discourses presented in them were devoted, according to J. Valsiner, "to social positioning of oneself within some general perspective designated by an-ism (mentalism, behaviorism, cognitivism, interactionism, transactionism, socioculturalism, and even humanism!) and setting up socially normative prescriptions for the methods through which "scientific facts" are produced. The-isms have been fighting one another for dominance in the field-leading from the "Era of Behaviorism" to the "Cognitivism Restoration," and to further eras" [23]. Time turned out to be the best healer from the unrealistic optimism of their representatives regarding the very possibility of alone universal exhaustive solutions finding. Behaviorists, the most consistent and orthodox builders of objective psychology, could not overcome the opposition to the activity of the black box that they ignored in every way, gradually evolving into a cognitive-scientific-behavioral approach with much smaller aplomb.

Such heterogeneity did not fit into the framework of onedimensional logic. Methodological foundations of the paradigm variety in the form of alternative ontological-epistemological dichotomies (objective-subjective, determinism-indeterminism, heredity-variability, cognition-unknowability, atomism-holism, rationalism-irrationalism, static-dynamic, ethic-emic, nomotheticidiographic, etc.), as well as the justification for the impossibility of their unification to a single basis, were presented in a particular study [2].

Something similar happened in the research areas, in which the personality, environment, and behavior (activity) were constituted from the time of Levin. The accent of each of them isolatedly led psychological knowledge into the methodological deadlock. Accent on the environment in the absence of personality and its activity led to the same deadlock. On the example of the activity approach, the same thing happened with the prioritization of activity and personal determination. In place of the Levin's non-directional determination, articulatedin theuniversalformula $\mathrm{B}=\mathrm{f}(\mathrm{P}, \mathrm{E})$ comesin partbi-directional determination of $\mathrm{A}$. Bandura, expressed in his well-known formula $\mathrm{B}$ $=\mathrm{f}(\mathrm{P} \rightleftarrows \mathrm{E})$, emphasizing the interdependent and mutually encouraging character of the personal relationship, environment, and activity.

G. Henriques, assessing the current state of the worldview that defines human activity and discourse, positions it as "fragmentary pluralism", characterizing the philosophical worldview as fundamentally contradictory and incompatible and as a constructive alternative offers integration pluralism, "where there are differences in emphasis that stem from disparate needs, goals, and other idiographic factors, but each individual is connected to the same, common base of shared, general understanding" [24].

Thus, it is in the dialogue of alternatives, including the paradigm level, that scientific revolutions clearly illustrate a unique opportunity of overcoming inevitable for any knowledge built on a universal, unchanging dogmatic ground, a deadlock situation characterized by the exhaustibility of its heuristic potential.

For the dialogue to take place, its subject matter is necessary. The difficulty of it formulating concerning psychological knowledge is due to the presence of a multitude of alternatives representing different approaches, traditions, paradigm coordinates and epistemologies, which have an extremely limited commonality. Apart from the abstract construct "psyche," evaluated as the basis of similarity, but also acting as the subject of non-alternative discussions, the areas of agreement are minimal.

The first solution that comes to mind is the formulation of definition including all traditions and approaches, considering the psychological phenomenology continuums described earlier, representing the nature's heteroqualitativity, psychic spheres, and behavior determinants. It is these grounds that determine the subject of psychology as "being-in-the-world of the Self as a biopsycho-symbolic socioculturally interdeterminated dialogic entity in interaction with the social and natural environment in consciousunconscious-existential dimensions" [25]. The category of "being-inthe-world" focuses attention on the existential aspect of a person's being, emphasizing its "interwovenness" in his life, experiencing it. The category "Self" concentrates on the aspect of the relationship between the internal and external, manifested in the relationship of the person with his external environment, history, etc. The threedimensional bio-psycho-social continuum focuses on the complexity of the three heteroqualitative natures interaction - biological, psychic, and social (symbolic). Sociocultural interdeterminism emphasizes the aspect of the personality, activity, and its situational context interdependence manifested in the fact that any change in one of them leads to change in the two others. Finally, phenomenology is historically analyzed in the dimensions of the conscious, unconscious, and existential, constituting yet another continuum of the mental health and wellbeing phenomenology comprehension.

The subject definition necessarily requires the consideration of the being analyzed phenomenology qualitative uniqueness, which I see in the following: 
1. lack of direct access to psychic reality, the impossibility of it is isomorphic and authentic measurement and verification;

2. the dominance of the second-sign or symbolic system, which allows us to break away from "here and now," being relocated in space and time;

3. heteroqualitative natures-biologic, psychic, and symbolic (social);

4. semiotic subjectivity and intersubjectivity;

5. the existential experience, interwovenness being-of-the-world;

6. the significant influence of the unconscious, which has been recorded and transformed in the experience;

7. cultural-historical conditioning;

8. active participation in the process of creating circumstances of one's own life [6].

It is this qualitative specificity that sets the limits in the field of ontological-epistemological basis, methodology, and methods of research adopted in natural science. And you can infinitely improve the technical and technological support of research, ensure absolute transparency of the procedure and empirical data, etc., but the objective preparation of living entities that are experiencing their being-in-theworld, being in an endless process of developing semiotic meanings and senses that form and reinterpret meanings and norms, radically changing their attitude to objects and events under the influence of objective and subjective factors, much more, but it will be impossible to reach an exhaustive result in principle. Killing the living body, you can get a tremendous anatomical picture. It will be all good only soulless. Therefore, scientific research requires qualitatively different tools and technologies based on adequate methodological grounds, free from a set of scientific inferiority around natural sciences. Moreover, if knowledge does not run away from real life, producing knowledge conducive to its harmonization, then its authority will be much higher than if it will drive itself into the standard frames and templates. Moreover, if in physics the object of theories verification is inanimate nature, then in psychology - living social life.

The definition in the subject as the initial basis requires elaboration of the conditions for the dialogue implementation. The first condition is the creation of an alternative to classical non-alternative logic or/ or in the form of dialogic logic and/and. In this logic, alternative approaches are beginning to be viewed not as mutually exclusive, but as complementary. The goal of scientific knowledge development becomes the development of ways and means for establishing productive inter-paradigm and interdisciplinary dialogue aimed at mutual enrichment and mutual development in the field of the psychological phenomenology understanding deepening. The practical implementation of this kind of logic requires the development of a fundamentally new methodological approach that allows us to create the basis for genuine dialogue that inherently assumes mutual acceptance and joint consensual type decisions development.

The author's attempt to solve this problem is presented in the framework of an integrative-eclectic approach to the psychological phenomenology analysis in the conditions of existing traditions diversity [2]. The essence of the approach is a multi-facet, multilinear, multi-vector analysis that creates the possibility of qualitatively different "insight," involving the inclusion in consideration of various aspects of multiplicity, dialogicity, diatropicity of the phenomenon under study. The ability to stand on the opponent's position, the inclusion of competitive ideas, critical reflection, critical positioning provides the possibility of an alien analysis, turning into yet another "perpetual motion" of the knowledge progress. This is not about integration, which inevitably generates a tendency to monopolize the truth with all ensuing consequences, namely, the free operation of multi-plane, multi-vector knowledge associated with the richest traditions in the problem area and their tools.

The development of the integrative-eclectic approach found its expression in a sociocultural-interdeterministic dialogical addendum that offers many conditions for the mental health and wellbeing phenomenology understanding deepening:

1. pluralism and tolerance in their essence, realized in the essential, conscious acceptance of the possible and useful existence of alternative explanations of the nature of the phenomena being analyzed;

2. alignment of the basic ontological-epistemological foundations that define the attitude toward the fundamental issues related to the knowability of the reality to be studied and explained, accompanied by an affirmation of the congruence and misalignment of positions;

3. sociocultural interdetermination, expressed in recognition of the reciprocal influence and reciprocal conditioning of all the factors present in the functioning of the phenomenon being studied;

4. dialogicality manifested in the ability to create shared knowledge that takes into account individual and cultural differences, based on the logic of mutual enrichment and mutual development [3].

Having determined the dialogues methodological and theoretical basis, it is possible to proceed to the question connected with the essence of proposed metatheory. The paramount question is the definition of the content framework of the theory or spaces of psychological phenomenology, on which it can be extrapolated. Orientation to the construction of the integrative property spaces presupposes definition in general basis that permeates all the others and causes their uniqueness.

The renewed interest for psychological phenomenology cultural conditioning served as the basis for the idea of a four-dimensional continuum, where culture acts as the fourth universal pervasive dimension. This resonates with the modern context of the Cultural Revolution in psychology [26]. Moreover, empirical evidence of the cultural conditioning (presence of pronounced cross-cultural differences) is accumulated in various areas of scientific knowledge (anthropology, biology, medicine, neurosciences, etc) [27-30].

A cultural-dialogical interdeterminist solution of the psychological knowledge integration problem. As the author's alternative, integrating the productive potential of both approaches, is offered dialogical interdeterminism [4]. Respectively, the opposition of determinism and indeterminism, being transformed to dialogical indeterminism, finds the new quality integrating their opportunities and leading for mutual enrichment and mutual development and, as a result, the finding of psychological phenomenology understanding deepening added resources. The prefix "inter" shows a way using which we can overcome a root metaphor of psychological understanding as at the public, and scientific levels, having kept them in an initial state. Such kind metaphor represents the distinction between internal/ external with external space comprehension. Meaning is neither "inside" nor "outside": it is "between" [31]. Meanings co-construction 
and handling are the processes organizing interaction including of psychic heteroqualitative natures and spheres. In interaction, there is (or does not occur) achievement of the peculiar mutually arranging compromise providing either the optimum or minimum enough condition of coexistence allowing behavior integration and coordination.

The particular question represents the relationship between the offered allocated structure elements. The most known attempts of this problem solution relate to names of outstanding metatheoristsK. Lewin [32] and A. Bandura [33]. In his Field Theory, K. Lewin describes the behavior as a function from the personality and an external environment $B=f(P, E)$. Characterizing K. Lewin's approach as unidirectional $\mathrm{A}$. Bandura offered partly directional transformation of its formula $\mathrm{B}=f(\mathrm{P} \rightleftarrows \mathrm{E})$, subsequently transforming it into the principle of reciprocal determinism, representing the interaction of elements as mutually directed. The co-rations of these approaches to the author's $4 \mathrm{D}$-directed interdeterminism is shown in Figure 2.

In the development of the presented approaches, I offered the principle of a dialogical interdeterminism $[4,6,7]$. The relation of dialogical interdetermination emphasizes an aspect of interaction interinfluencing and mutually changeable character. Any change of heterogeneous, nonlinear, dynamic system one elements inevitably leads also to change in all interconnected elements. Moreover, occurred changes lead to change of the most dynamic heterogeneous system's quality receiving new growths in the form of expansion, reconsideration, and re-experience of the found experience. These elements at the same time are both autonomous and mutually causing each other and the last focus attention that each of the elements does not exist as self-sufficient but only in relationship with others.

In the process of sociocultural-interdeterminist dialogue behavior, internal personal factors and the environment influences are interdependent interdeterminants of each other, conditioned by interdeterministic interactions of heteroqualitative biological, psychic, and social (symbolic) natures, and spheres of conscious, unconscious, and existential, conditioned by the context of culture in foreshortening of personality, environment, and activity. By the introduced principle of dialogical interdeternism, structural elements of the offered continuums are in a condition of interdeterminist interaction. Changes of continuum one element lead to change of the most dynamic heterogeneous system quality receiving new growths in the form of reconsideration and re-experience of the found state.

A distinctive feature of the offered approach is the articulation of the dialogical nature of the interaction between elements of the heterogeneous system. The initial is Bakhtinian reading of the dialogue as an interaction in conditions of a multi-voiced environment, assuming the unconditional acceptance of Otherness and differentness, accompanied by orientation toward the compatibility development, mutually accepted balance and sharedness [34]. It is the dialogue that can act as a mechanism for interdetermination of heteroqualitative natures, psychic spheres, and behavioral determinants, ensuring finding homeostatic property balanced results.

The discussed problems consideration in the context of dialogic interdetermination should be carried out from the standpoint of dialogical thinking. J. Salgado and J.W. Clegg distinguish six fundamental principles of dialogical thinking: "the principles of relationality, dynamism, semiotic mediation, alterity, dialogicality, and contextuality. Together, these principles imply a notion of psyche that is neither an isolated homunculus nor a disembodied discourse, but is, rather, a temporally unique, agentive enactment that is sustained within, rather than against, the tensions between individual and social, material and psychological, multiple and unified, stable and dynamic" [35].

Dialogism focuses on change and its regulation emphasizing that dynamic relation is the essential constant in, and the basis for, existence. Extending this to the human context, according to Markova's [36] reasoning's, existence implies a relationship between an Ego and an Alter. Dialogism does not dissolve the person into the social realm; it assumes, instead, that personal agency has a fundamental role in

\section{Undirectional (K. Levin, 1936) $B=f(P, E)$}

Partly directional (A. Bandura, 1978) $B=f(P \leftrightharpoons E)$

Reciprocal (A. Bandura, 1978)

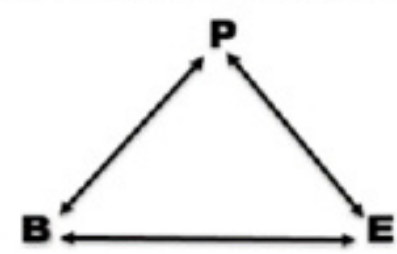

4D-directional (V. Yanchuk, 2014)

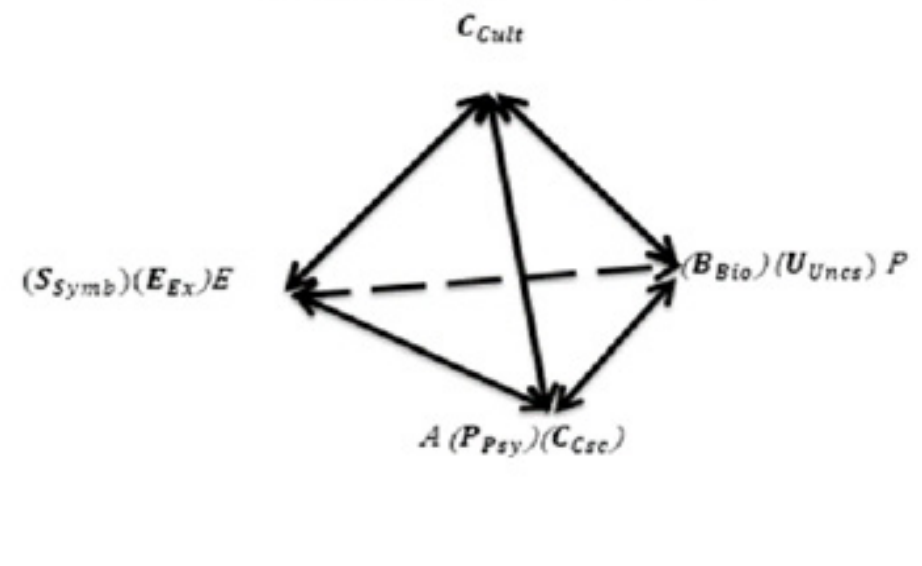

\section{Where: $\mathrm{P}$ - personality; $E$ - external environment (situation): $\mathrm{B}$ - behavior}

Where: $A$ - activity, $P$ - personality, $E-$ environment,
$\boldsymbol{B}_{B i o}-$ biological; $\boldsymbol{P}_{P s y}-$ psychic; $\boldsymbol{S}_{S y m b}-$ symbolic;
$\boldsymbol{C}_{C s c^{-}}-$conscious; $\boldsymbol{U}_{U n c s}-$ unconscious; $\boldsymbol{E}_{E x}-$ existential;
$\boldsymbol{C}_{C u t t}-$ cultural-historic context.

Figure 2: Comparison of unidirectional [32], partly directional, reciprocal [33], and 4D-directional approaches for behavior determination. 
determining human thought, action, and experience. A vital element of these processes for psychiatry is the intersubjective grounding of experience through modes of embodied interpersonal interaction, cooperation, and collaboration [37]. The dialogicity on its nature is interdeterministicsince assumes the interaction leading to mutual changes and mutual development, in turn leading to the new qualities finding which are not presented in an initial state of the dynamic heterogeneous system interacting elements. At the same time, the acquisition of this new quality, which is a joint achievement, leads to the broader relationships system stabilization through certainty in them by acquiring deterministic influence.

The relation of dialogical interdetermination emphasizes the element of the mutually influencing and mutually changing nature of it. Any change in one of the elements inevitably leads to change in all interrelated elements. Moreover, the changes that have occurred lead to change in the quality of the dynamic heterogeneous system itself, which receives growth in the form of expansion, rethinking, and re-overcoming the acquired experience. These elements are both autonomous and mutually reinforcing, and the latter focuses on the fact that each of the elements does not exist as self-sufficient, but only in relationship with others.

At the same time, the very concept of interaction is exceptionally restrictive about co-existence or heterogeneous entities dialogue that does not acquire newly integrated quality but retains their original uniqueness but forms new quality that is outwardly above its constituents. The most authentic for describing this state is the concept of dialogue in its Bakhtinian understanding [34]. When people are in a dialogue, they interact through mutually active dynamics of clarifying discourses like forming meanings and agree on the needs, interests, and desires that will be achieved in the event of agreement, while maintaining their uniqueness and relative autonomy. The dialectical nature of the interdeterministic interaction is manifested, firstly, in the unconditional acceptance of the Other, caused by the impossibility of an incompatible existence in principle, and secondly, acquiring of new quality by the interacting parties that are not present in either of them individually. This specific character of dialogue as a form of interaction is also conditioned by the qualitative specificity of the man himself as an object and subject of cognition. Bakhtin stresses in this connection that that subject as such cannot be perceived and studied as a thing, for as a subject he cannot become a subject, being mute. Therefore his cognition can only be dialogic [34]. In general, the following features can characterize M. Bakhtin's dialogism, systematized J. Salgado и J.W. Clegg:

1. the primacy of relations over entities (relationality);

2. that relations are dynamic and developing processes (dynamism);

3. that human relations are mediated by signs (semiotic mediation);

4. that a relationship implies alterity, that is, a relationship between I and Other (alterity);

5. that human relationships are dialogical, or negotiated, relationships (dialogicality);

6. that dialogical relationships include and depend upon a sociocultural context (contextuality) [35].

The authors emphasize that "dialogism conceives of human beings as beings-in-relation-with-others, it conceptualizes ongoing experience regarding the dynamic negotiations that constitute such relations. It is through (and by) communicative relation that meaning is brought to being ... The negotiation of meaning involves a vast array of voices brought to bear in particular languages, social norms, personal and social histories, and other forms of shared meaning ...Dialogism makes the claim that the psyche and its relational, sociocultural context are inseparable (the principles of relationality and contextuality) but non-identical (the principle of alterity)" [38].

The listed features of dialogism provide the basis for integrating the heteroqualitative nature, psychic spheres and behavior determinants of psychological phenomenology that are covered in the proposed meta-theory, represented as three culturally conditioned fourdimensional spaces: biological-psychic-symbolic, consciousunconscious-existential and personality-environment-activity. Each of the components of the allocated spaces is in the relation of dialogical interdetermination, forming in aggregate a single dynamic heterogeneous systemic whole or quality that is not reducible to a simple sum of its constituents. Thus, the dialogue of spaces takes the form of a pyramid, each element of which is equivalent, as shown in Figure 3.

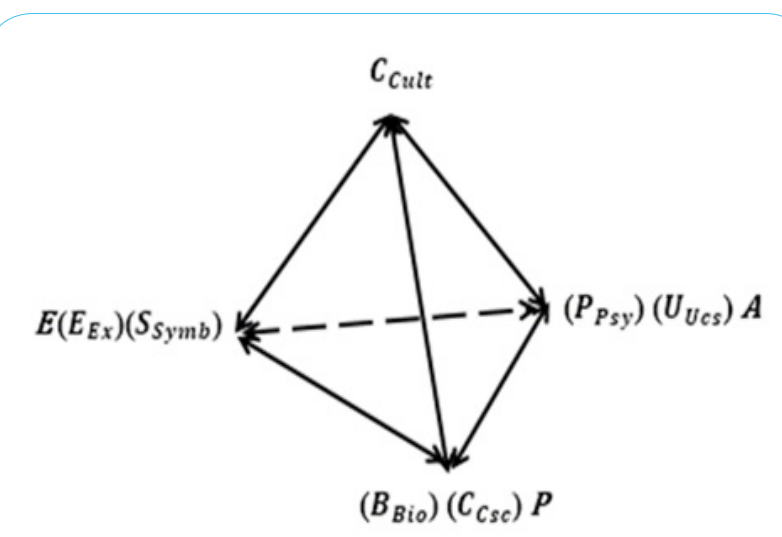

Figure 3: The state of dialogic interdetermination. Where: $C_{C u l t}{ }^{-}$ Cultural development; $B_{B i o}$ - biological; $P_{P s y}$ - psychic; $S_{S i m b}$ - symbolic; $C_{C s c}{ }^{-}$conscious; $U_{U n c s}$ - unconscious; $E_{E x}$ - existential; A - activity; P - personality; E - environment.

The preceding allowed us to expand the framework of the principle of reciprocal determinism qualitatively by focusing attention, firstly, on the interdeterminative nature of the interaction of personality, situation, and activity (behavior) determinants, and, secondly, considering their cultural conditionality. Here we are not talking about the state of unidirectional or partially bi-directional determination, but the qualitatively distinctive character of the dialogic property interaction, which assumes the initial equivalence, interdependence, and mutual influence, leading to the new qualitative state formation not represented in separate determinants.

As examples of the dialogical interdetermination productive use in the sphere of heteroqualitative natures, we may cite the intensification of research in the field of the biopsychosocial model application in medicine and medical psychology [39], psychic spheres - Freudian catharsis in psychoanalysis, behavior determinants successful examples of cultural integration [40]. A relatively extensive amount of empirical material has been accumulated on this kind of interdetermination concerning biological knowledge. Numerous studies show the adaptive transformation of brain structures to conditions of interaction with the environment [41], genetic changes [42] related to changes in the natural environment (e.g., environmental warming and pollution) and many other results. 
Applied possibilities of cultural-dialogical interdeterminist metatheory. With the metatheory seeming abstractness, it has practically unlimited applications in all areas of psychological knowledge.

Moreover, recent studies clearly demonstrate the need and prospects for focusing on new qualities that are determined by the interaction of the constituent elements [43-46]. Today is more evidence that the relationship of mechanisms to symptoms is many-to-many - that is, most neurobiological perturbations give rise to multiple symptoms, and any given symptom can result from more than one underlying mechanism (though there may be a final common pathway). Moving from symptoms to syndromes makes the relationships still more complicated, requiring dynamic system models [47]. Understanding cultural influences on the mechanisms of psychopathology requires a conceptual shift away from neurobiological reductionism toward an eco-social view of the mind as embodied, embedded, and extended [48]. Recent epigenetic studies have highlighted the crucial role of environments in gene expression and brain development. These studies suggest that interrelated bio-psycho-social channels involved in the development of chronic physical aggression are generally the product of an intergenerational transmission process occurring through assortative mating, genetic inheritance, and the inheritance of physical and social environmental conditions that handicap brain functioning and support the use of physical aggression to solve problems. Given these intergenerational mechanisms and physical aggression on set in infancy, it appears clear that preventive interventions should start early in pregnancy, at the latest [49].

In J.F. Rauthmann and colleagues researches [50] were established three basic principles of environmental (situational) determination of behavior psychological study: processing, reality, and circularity. The processing principle is concretized in that the process of processing information about the situation is crucial for determining it and affects the individuals in it. This means that the situation affects thinking, feelings, desires, and actions in the process of mental processing of information about it. The reality principle clarifies how different realities - physical (signals), consensual reality (normative social reality) and idiosyncratic reality (unique personal reality) - are contained in the situation and agree upon objective and subjective perspectives ...The principle of circularity draws attention to the fact that personal perceptions and characteristics of the situation itself are combined in the case of determining and measuring the situation in definitions (i) mental states or behavior (state assessment); (ii) assessment or conclusions about the effects of mental state or behavior (impact assessment); and/or (iii) only their own perceptions (approximation assessment) [50].

A demonstration of the dialogic interdetermination specifics was shown on the example of the bio-psycho-social adaptation of an alcoholic'sphenomenon.Itisasociocultural-interdeterministicdialogic process of harmonizing natural, biological, and psychological states, socio-cultural dispositions, aimed at achieving balance (physiological, psychological, existential) in interaction with the immediate and mediated social and natural environment. Moreover, the situation is considered in the context of complex interdeterministic interaction of bio-psycho-social or natural, psychic, and symbolic properties. The study shows that overcoming alcohol dependence presupposes not only exemption from alcoholic biochemical dependence, but also the formation of the eco-cultural dialogic supportive environment (selfhelp groups, family-supporting environment that helps the formation of a new quality of a person independent from alcohol, increasing the ability for self-determination, etc.), that promote social rehabilitation. It also presupposes deep psychological work with existential problems, associated with the awareness of their defectiveness and social rejectionness and formation ability for alcohol-independent self-determinated behavior. Formation of this kind self-determinated behavior is causally related to resolving unconscious conflicts through psychoanalytic procedures. Thus, the possibilities of involving conscious-unconsciously-existential space to the alcohol-dependent patients psychological problems solution significantly increases the effectiveness of rehabilitation intervention.

The role of cultural conditioning of different-quality natures and areas of psychological phenomena research was clearly demonstrated in the cross-cultural comparison of Iranian and Belarusian patients experiencing chronic pain [51]. The study shows the critical role of psychosocial factors in the results of pain experience that determine the specific behavioral response of the patient to the perception of physiological disorders. It is shown that the role of psychological and social factors increases in comparison with biological factors as the pain becomes more chronic [38]. An embodiment of this idea in practice is the emergence of a new direction in medicine biopsychosocial medicine [52]. It was clearly demonstrated, that in clinical practice, attention to culture serves multiple functions: (1) it can enable patients to communicate their concerns in ways that are experience-near and meaningful to themselves and others in their family and community; (2) it can help clinicians interpret the diagnostic significance of symptoms and behaviors and assess patients' predicaments in relation to relevant norms and contexts; (3) it can guide the development of culturally appropriate treatment plans and interventions; and (4) it is essential for negotiating the delivery of interventions and assessment of outcomes [53].

Without intending to further argue the perspectives of the proposed metatheoretical approach for discussion of problem individualism collectivism, I note that, in its framework, special attention must be paid for results of heteroqualitative natures, psychic spheres and areas of their manifestation interaction. In the context of integrative new quality, in particular, interest is constructed that denote the results of such dialogical interdeterminative interaction, which include inter subjectivity, intertextuality, and interexistentiality. All these constructs have a dual nature - they determine interaction results, conditions for its success and grounds and prospects for subsequent development.

\section{Discussion}

Summarizing above, I emphasize once again the need to broaden the horizons of the problem of mental health and well-being vision by finding additional resources for its phenomenology understanding through: first, giving them the character of multidimensionality, multi paradigm ality, and multidisciplinarity, involving the applying knowledge resources, accumulated in the conjugate psychological knowledge paradigm coordinates systems and related disciplinary fields. Second, awareness in the cultural-dialogic-interdeterministic character of heterogeneous, nonlinear, anddynamic indeterminant's functioning. Third, the culturezation of the mental health and well-being phenomenology through the involvement of the current cultural psychology conceptual apparatus. Fourth, going beyond personocentrism by including in the analysis plane the interdeterminative influence of the ego-involvement, including the social, natural and technogenic environment, placed in a specific historical socio-cultural context, etc. 
Characterizing the presented metatheoretical approach in general, we can state the following:

1. The starting point is the statement of the complex bio-psychosocial culturally conditioned heterogeneous nonlinear dynamic nature of mental health and well-being phenomenology. Awareness that many personalities mental disorders, psychiatric and psychological problems are associated with biological disorders manifested in mental activity disorders and, conversely, many of the biological level dysfunctions have a psychological underpinning (a glaring example of which are numerous psychosomatic disorders). Both these and others are often provoked by social disharmony (and, in turn, determine it), which is actualized in interpersonal contradictions and related interpersonal and intrapersonal conflicts, which cause discomfort in social interaction and, as a result, dissatisfaction with oneself. Finally, there are marked cultural differences in each of the constituents that need to be considered, especially in intercultural interaction.

2. The biological -psychic- the symbolic (social), as well as the conscious - unconscious - existential, personality - environment and the activity, are in a state of dialogic interdetermination, manifested in their interdependence and mutual influence, expressed in acquiring a new quality not reducible to the simple sum of its constituent parts. A qualitative change in one element inevitably leads to changes in designated heterogeneous system interconnected elements, and vice versa. The acquiring of dynamic heterogeneous system new quality presupposes the preliminary preparation of each of the elements for future qualitatively new state, including the bio-psycho-social and cultural preparedness formation, awareness of the new state uniqueness and the potential changes associated with its acquisition, preventive correction of potential unconscious contradictions and inconsistencies fixed in the experience of the old new, contributing to the existential acceptance of the altered self in his being-in-the-world and recognizing himself as a selfinterdependent agent.

3. The optimal state of the dynamic heterogeneous system is provided through the dialogue of heteroqualitative natures, psychic spheres, and behavior determinants, ensuring the holistic functioning balance, by finding and mutually developing mutually acceptable compromises and co-existence optimums. Once this balance is broken, various disharmonies and dysfunctions begin to manifest, stimulating the search for overcoming resources. Specificity of the dialogical form of interaction in dynamic heterogeneous system is manifested in the unconditional acceptance of the Otherness of co-participants in the process of joint functioning, orientation toward finding mutually reinforcing solutions, formation of special kind bio-psycho-symbolic, consciously-unconsciously-existential homeostasis, creating common basis for coordinated and synchronized mutual understanding and mutual development in the context of specific social and natural environment and being carried out activities.

The preceding allows us to formulate the following perspectives of mental health and wellbeing phenomenology research:

1. Need for the phenomenology of mental health and wellbeing consideration in the aspects of heteroqualitative natures (biological - psychic - symbolic), spheres of psychic (conscious
- unconscious - existential) and research areas (personality external environment - activity)in the context of their cultural conditioning.

2. Phenomenology of mental health and well-being must be analyzed in the context of heterogeneous nonlinear dynamic systems elements dialogical interaction considering acquisition by system elements new quality, not reducible to the simple sum of constituent parts.

3. Cultural-dialogic interdetermination presupposes interaction aimed for achieving a mutually acceptable state of balance that contributes to the heterogeneous nonlinear dynamic system optimal functioning achievement at the level of heteroqualitative natures, psychic spheres, and behavioral determinants.

In general, the cultural-dialogical interdeterministic approach to the mental health and wellbeing phenomenology allows providing a comprehensive solution to widespread range problems that modern society faces.

\section{Competing Interests}

The authors declare no competing interests.

\section{References}

1. Kirmayer L, Gomez-Carillo A (2019) Culturally Responsive Clinical Psychology and Psychiatry: An Ecosocial Approach. In Cultural Clinical Psychology and PTSD.

2. Yanchuk V (2000) Methodology, theory and the method in psychological phenomenology analysis: An integrative-eclectic approach. Minsk: Bestprint.

3. Yanchuk V (2012) Interparadigm dialog as the resource of psychological phenomenology understanding deepening: sociocultural-interdeterminist dialogic perspective. Psychological Journal 1-2: 4-17.

4. Yanchuk V (2018b) Cultural-dialogical metaperspective of psychological knowledge integration in conditions of uncertainty and constructivist diversity. Methodology and History of Psychology.

5. Yanchuk V (2016) Sociocultural-interdeterminist dialogical metatheory of psychological knowledge integration. International Journal of Psychology 51: 438-439.

6. Yanchuk V (2018a) The epistemological potential of the construct "culturaldialogic interdeterminism. History and philosophy of science in the time of change. Moscow: Russian society of the history and philosophy of science.

7. Yanchuk V (2018c) The Theoretical and Empirical Foundations of the Sociocultural-Interdeterminist Dialogical Metatheory of the Integration of Psychological Knowledge. Journal of Russian \& East European Psychology 55: 241-286.

8. Eronen M (2009) Reductionist Challenges to Explanatory Pluralism: Comment on McCauley. Philosophical Psychology 22: 637-646.

9. Gergen K (2009) Pragmatics and Pluralism in Explaining Human Action. Behavior and Philosophy 37: 127-133.

10. Goertzen J (2010) Dialectical pluralism: a theoretical conceptualization of pluralism in psychology. New Ideas in Psychology 28: 201-209.

11. Healy P (2012) Toward an integrative, pluralistic psychology: On the hermeneutico-dialogical conditions of the possibility for overcoming fragmentation. New Ideas in Psychology: 30: 271-280.

12. Lazard L, Capdevila R, Roberts A (2011) Methodological Pluralism in Theory and Practice: The Case for $\mathrm{Q}$ in the Community. Qualitative Research in Psychology 8: 140-150.

13. Mendelowitz E, Kim CY (2010) William James and the Spirit of Complexity: A Pluralistic Reverie. Journal of Humanistic Psychology 50: 459-470.

14. Redding RE (2001) Sociopolitical Diversity in Psychology: The Case for Pluralism. Am Psychol 56: 205-215.

15. Smythe $W E$, McKenzie SA (2010) A vision of dialogical pluralism in psychology. New Ideas in Psychology 28: 227-234. 
Citation: Yanchuk VA (2019) Cultural-Dialogic Interdeterminist Metaperspective for Mental Health and Well-Being. Int J Psychol Behav Anal 5: 160. doi: https:// doi.org/10.15344/2455-3867/2019/160

Page 10 of 10

16. Watanabe T (2010) Metascientific foundations for pluralism in psychology New Ideas in Psychology 28: 253-262.

17. Paterson B (2008) Pluralism. The Sage Encyclopedia of Qualitative Research Methods. London, UK: SAGE Publications.

18. Lilienfeld S (2017) Psychology's Replication Crisis and the Grant Culture: Righting the Ship. Perspectives on Psychological Science 12: 660-664.

19. Fogel A, Lyra CDP, Valsiner J (1997) Dynamics and Indeterminism in Developmental and Social Processes. Psychology Press.

20. Valsiner J, Molenaar P, Lyra M, Chaudhary N (2009) Dynamic Process Methodology in the Social and Developmental Sciences. Springer.

21. Van Geert P (1997) Que Sera, Sera: Determinism and Nonlinear Dynamic Model Building in Development. Dynamics and Indeterminism in Developmental and Social Processes. Psychology Press.

22. Seligman R, Choudhury S, Kirmayer $\amalg$ (2016) Locating culture in the brain and the world: From social categories to the ecology of mind. The Oxford Handbook of Cultural Neuroscience. Oxford, UK: Oxford University Press.

23. Valsiner J (2009) Integrating Psychology within the Globalizing World: A Requiem to the Post-Modernist Experiment with Wissenschaft. IPBS: Integrative Psychological and Behavioral Science 43: 1-21.

24. Henriques $G$ (2008) The Problem of Psychology and the Integration of Human Knowledge: Contrasting Wilson's Consilience with the Tree of Knowledge System. Theory \& Psychology 18: 731-755.

25. Yanchuk $V$ (2006) The postmodernist, sociocultural-interdeterminist dialogism as the prospect of positioning in the psychology subject. Methodology and History of Psychology. Issue 1: 193-200

26. Valsiner J (2014) An Invitation to Cultural Psychology. NY: SAGE Publications.

27. Ellis BD, Stam HJ (2015) Crisis? What crisis? Cross-cultural psychology's appropriation of cultural psychology. Culture \& Psychology 21: 293-317.

28. Keith K (2011) Cross-Cultural Psychology: Contemporary Themes and Perspectives. Wiley-Blackwell.

29. Teo T (2018) Outline of Theoretical Psychology: Critical Investigation. London: Palgrave Macmillan.

30. Gelfand NG, Chi-yue C, Ying-yi H (2011-2017) Advances in Culture and Psychology. New York: Oxford University Press.

31. Mininni G (2010) The Method of Dialogue: Transaction Through Interaction. Integr Psychol Behav Sci 44: 23-29.

32. Lewin K (1934) Principles of topological psychology. New York: McGraw-Hil.

33. Bandura A (1978) The Self-System in Reciprocal Determinism. American Psychologist 33: 345-358.

34. Bakhtin M (1973) Problems of Dostoevsky's poetics (R.W. Rotsel, Trans.). Ann Arbor, MI: Ardis.

35. Salgado J, Clegg JW (2011) Dialogism and the psyche: Bakhtin and contemporary psychology. Culture \& Psychology 17: 421-440.

36. Markova I (2003) Constitution of the self: Intersubjectivity and dialogicality. Culture \& Psychology 9: 249-259.

37. Fuchs T, De Jaegher H (2009) Enactive intersubjectivity: participatory sensemaking and mutual incorporation. Phenomenology and the Cognitive Sciences 8: 465-486.

38. Smythe WE, McKenzie SA (2010) A vision of dialogical pluralism in psychology. New Ideas in Psychology 28: 227-234.

39. Hatala A (2012) The Status of the "Biopsychosocial" Model in Health Psychology: Towards an Integrated Approach and a Critique of Cultural Conceptions. Open Journal of Medical Psychology 1: 51-62.

40. Powers JT, Cook JE, Purdie-Vaughns V, Garcia J, Apfel N, et al. (2016) Changing Environments by Changing Individuals: The Emergent Effects of Psychological Intervention. Psycholo Sci 27: 150-160.

41. Gergen K (2010) The acculturated brain. Theory \& Psychology 20: 795-816.

42. De Jong $\mathrm{H}$ (2000) Genetic determinism. How not to interpret behavioral genetics. Theory \& Psychology 10: 615-637.

43. Balliet D, Tybur JM, Lange PAM (2017) Functional Interdependence Theory: An Evolutionary Account of Social Situations. Pers Soc Psychol Rev 2: 361368.

44. Grant JG, Savage T, Friedman LH (2015) International "Best Practices" in Health Care: The Roles of Context and Innovation. Adv Health Care Manag 17: $31-35$.
45. Posner MI, Ruthbart MK (2017) Integrating brain, cognition, and culture Journal of Cultural Cognition 1: 3-15.

46. Suls J, Rothman A (2004) Evolution of the Biopsychosocial Model: Prospects and Challenges for Health Psychology. Health Psychology 23: 119-125.

47. Cramer $\mathrm{AO}$, van Borkulo CD, Giltay EJ, van der Maas HL, Kendler KS, et al. (2016) Major depression as a complex dynamic system. PloS One.

48. Kirmayer LJ, Ramstead MJ (2017) Embodiment and enactment in cultural psychiatry. In T. F. C. Durt, Embodiment, enaction, and culture: Investigating the constitution of the shared world. Cambridge, MA: MIT Press.

49. Trembley RE, Vitaro F, Cote SM (2018) Developmental Origins of Chronic Physical Aggression: A Bio-Psycho-Social Model for the Next Generation of Preventive Interventions. Annual Reviews 69: 383-407.

50. Rauthmann JF, Sherman RA, Funder DC (2015) Principles of Situation Research: Towards a Better Understanding of Psychological Situation. European Journal of Personality 29: 363-381.

51. Goli Z, Yanchuk V (2012) Effect of Racial and Ethnical Differences in Pain Perception: Explaining the Effective Mechanisms on It. Psychological Journal 3-4: 41-50.

52. Junne F, Zipfel S (2015) Research prospects in BioPsychoSocial medicine: new year reflections on the "Cross-Boarder Dialogue" paradigm. BioPsychoSocial Medicine 9: 1-4.

53. Kirmayer L, Swartz L (2014) Culture and global mental health. In M. P. V. Patel, Global mental health: Principles and practice. Oxford, UK: Oxford University.

54. Yanchuk V (2014) Sociocultural-Interdeterminist Dialogical Perspective of Intercultural Mutual Understanding Comprehension Deepening. Open Journal of Social Sciences 2: 178-191. 